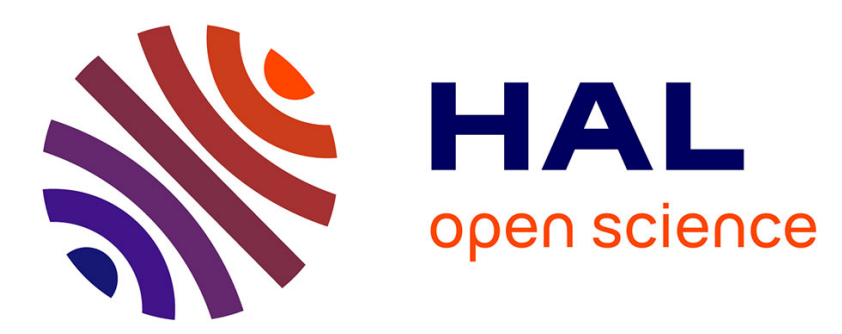

\title{
Control of a Wave Equation with a Dynamic Boundary Condition
}

\author{
Nicolas Vanspranghe, Francesco Ferrante, Christophe Prieur
}

\section{To cite this version:}

Nicolas Vanspranghe, Francesco Ferrante, Christophe Prieur. Control of a Wave Equation with a Dynamic Boundary Condition. CDC 2020 - 59th IEEE Conference on Decision and Control, IEEE, Dec 2020, Jeju Island (virtual), South Korea. pp.652-657, 10.1109/CDC42340.2020.9303767 . hal02987252

\section{HAL Id: hal-02987252 https://hal.science/hal-02987252}

Submitted on 3 Nov 2020

HAL is a multi-disciplinary open access archive for the deposit and dissemination of scientific research documents, whether they are published or not. The documents may come from teaching and research institutions in France or abroad, or from public or private research centers.
L'archive ouverte pluridisciplinaire HAL, est destinée au dépôt et à la diffusion de documents scientifiques de niveau recherche, publiés ou non, émanant des établissements d'enseignement et de recherche français ou étrangers, des laboratoires publics ou privés. 


\title{
Control of a Wave Equation with a Dynamic Boundary Condition
}

\author{
Nicolas Vanspranghe, Francesco Ferrante, Christophe Prieur
}

\begin{abstract}
The general problem of this paper is the analysis of wave propagation in a bounded medium where the uncontrolled boundary obeys a coupled differential equation. More precisely, we study a one-dimensional wave equation with a nonlinear second-order dynamic boundary condition and a Neuman-type boundary control acting on the other extremity. A generic class of nonlinear collocated feedback laws is considered. Hadamard well-posedness is established for the closed-loop system, with initial data lying in the natural energy space of the problem. Moreover, we investigate an example of stabilization through a proportional controller.
\end{abstract}

\section{INTRODUCTION}

The aim of this paper is to study a wave equation in a bounded one-dimensional medium supplied with a dynamic (or kinetic) boundary condition at one end. The system is actuated via a Neuman-type control at the other end. Dynamic boundary conditions involve second-order time derivative and are typically obtained in physical models for which the momentum of the boundary cannot be neglected. A prime example is an infinite-dimensional model for the propagation of mechanical vibrations along drilling rods. In that case, the control is the torque applied to one extremity, and the kinetic boundary condition is given by the behavior of the rock-tip contact, which is subject to nonlinear friction. In particular, stick-slip phenomena can occur at the rock-tip interface and generate unwanted vibrations that might jeopardize the plant. A mechanical analysis of the rock-tip dynamics is given in [7]. The problem of stabilization and regulation of the velocity at the rock-tip contact has sparked interest in the control commmunity, see e.g. [10]. In engineering applications, the goal is to minimize the stick-slip vibrations through a suitable control. Various boundary control strategies are proposed to address this problem in [8], including backstepping design - see also [9]. In [4], an observer-based boundary control design is proposed. In [13], stabilization and regulation using a proportional integral boundary controller is investigated. In [12] and [1], a similar problem is considered, but with a boundary anti-damping only involving first-order derivatives. However, the aforementioned papers deal with linearized models. We should also mention [3] and [6] where different classes of boundary nonlinearities for distributed parameter systems are considered.

Let us introduce the specific dynamical model under study in this paper. Let $L>0$ and $\Omega \triangleq(0, L) \subset \mathbb{R}$. We deal with

Nicolas Vanspranghe, Francesco Ferrante and Christophe Prieur are with Univ. Grenoble Alpes, CNRS, Grenoble INP, GIPSA-lab, 38000 Grenoble, France. Email: name.surname@gipsa-lab.fr.

This work has been partially supported by MIAI @ Grenoble Alpes (ANR-19-P3IA-0003). the following control system:

$$
\begin{cases}\partial_{t t} u-\partial_{x x} u=0 & \text { on } \Omega \times \mathbb{R}^{+}, \\ \partial_{t t} u(0, t)-\partial_{x} u(0, t)=F\left(\partial_{t} u(0, t)\right) & \text { for all } t, \\ \partial_{x} u(L, t)=U(t) & \text { for all } t,\end{cases}
$$

where $F$ is a scalar function that models the nonlinear boundary friction, and $U(t)$ is the control input.

Our first goal is to prove the well-posedness of the control system (1) supplied with the following collocated feedback:

$$
U(t)=-g\left(\partial_{t} u(L, t)\right),
$$

where $g$ is a continuous nondecreasing scalar function. The output considered here is the velocity at the boundary where the actuator lies, meaning that an observer is not required to implement the controller. Note that this class of feedback laws includes nonlinearities of interest in control applications, such as saturations or deadzones - see e.g. [5].

In this paper, we prove that, under appropriate assumptions, closing the loop in (1) with (2) leads to a wellposed dynamical system. A precise variational formulation of the problem is given and the regularity of the solutions is rigorously investigated. The underlying control problem is the stabilization of a possibly non-dissipative boundary by an action on the other boundary. We prove that, under suitable conditions, exponential stability can be achieved using a proportional controler. To the best of our knowledge, the stabilization of such system in the presence of nonlinear boundary anti-damping has not been investigated so far.

This paper is organized as follows. Section $\Pi$ introduces the variational formulation of control system (1) and contains the first main result, namely the well-posedness of the closedloop dynamics. Section IIII introduces the natural energy associated with (1) and states an exponential stability result under appropriate assumptions on the nonlinear map $F$. This is the second main contribution. Section IV contains some illustrative numerical results. Section $\mathrm{V}$ gives the proof of the well-posedness result. Section VI contains concluding remarks.

Notation: Given a Banach space $E$, we denote its norm by $\|\cdot\|_{E}$ and we use the duality bracket $\langle\phi, x\rangle_{E}$ to write $\phi(x)$ for any $x \in E$ and $\phi \in E^{\prime}$, where $E^{\prime}$ is the topological dual of $E$. If $E$ is also a Hilbert space, its scalar product is written $(\cdot, \cdot)_{E}$. The space of infinitely differentiable functions on $\Omega$ with compact support is denoted by $\mathcal{C}_{c}^{\infty}(\Omega)$. Also, for $T>0$, we denote by $W^{1, p}(0, T ; E)$ the subspace of $L^{p}(0, T ; E)$ composed of (classes of) $E$-valued functions $f$ such that, for some $g$ in $L^{p}(0, T ; E), f(t)=f(0)+\int_{0}^{t} g(s) \mathrm{d} s$ for a.e. $t$ in $(0, T)$. Such class $f$ is identified with its continuous 
representative and we say that $f^{\prime}=g$ in the sense of $E$ valued distributions.

\section{VARIATIONAL FORMULATION AND WELL-POSEDNESS}

In this section, we establish the framework in which we analyze system (1), and state our well-posedness result. We start by introducing the energy spaces associated with (1) as well as some notation. First, define

$$
H \triangleq L^{2}(\Omega) \times \mathbb{R}
$$

We endow $H$ with the usual product Hilbertian structure. Define now the following subspace of $H$ :

$$
V \triangleq\left\{(u, u(0)): u \in H^{1}(\Omega)\right\}
$$

which is equipped with the scalar product inherited from $H^{1}(\Omega) \times \mathbb{R}$. As stated in Section $\mathbf{V}, V$ is also a Hilbert space. We also introduce the state space

$$
\mathcal{X} \triangleq V \times H
$$

endowed with the product Hilbertian structure. For the sake of clarity, we use parenthesis to denote elements of $V$ or $H$, and brackets to denote elements of $\mathcal{X}$, as in $\mathbf{X}=[\mathbf{u}, \mathbf{v}] \in \mathcal{X}$, $\mathbf{u}=(u, u(0)) \in V$, etc. Now, let us define the bilinear continuous symmetric form $a$ on $V \times V$ by

$$
a\left(\mathbf{u}_{1}, \mathbf{u}_{2}\right) \triangleq \int_{\Omega} \partial_{x} u_{1}(x) \partial_{x} u_{2}(x) \mathrm{d} x .
$$

Finally, we denote by $\delta_{L}$ the linear form mapping $\mathbf{u} \in V$ into $u(L)$, which belongs to $V^{\prime}$ since $H^{1}(\Omega)$ is continuously embedded into $\mathcal{C}(\bar{\Omega})$ in the one-dimensional case.

Assumption 1. The scalar function $F$ is globally Lipschitz.

We define the nonlinear operator $B$ on $H$ associated with the first-order boundary pertubation:

$$
\forall \mathbf{v}=(v, \gamma) \in H, \quad B(\mathbf{v}) \triangleq(0, F(\gamma)) .
$$

This operator is globally Lipschitz by Assumption 1, Let us precise the meaning of weak solutions to (1).

Definition 1. A weak solution to (1) on $(0, T)$ is any $\mathbf{u}$ in $L^{\infty}(0, T ; V) \cap W^{1, \infty}(0, T ; H)$ verifying

$$
\begin{aligned}
\int_{0}^{T} & -\left(\mathbf{u}^{\prime}(t), \phi^{\prime}(t)\right)_{H}+a(\mathbf{u}(t), \phi(t)) \mathrm{d} t= \\
& \int_{0}^{T}\left(B\left(\mathbf{u}^{\prime}(t)\right), \boldsymbol{\phi}(t)\right)_{H}+U(t)\left\langle\delta_{L}, \boldsymbol{\phi}(t)\right\rangle_{V} \mathrm{~d} t
\end{aligned}
$$

for all test-functions $\phi$ in $\mathcal{C}_{c}(0, T ; V) \cap \mathcal{C}_{c}^{1}(0, T ; H)$.

Definition 1 is motivated by simple formal calculations in which one multiplies the wave equation by some smooth test-function $\phi(x, t)$ and integrates it over $\Omega \times(0, T)$ using integration by parts and the boundary conditions. Note that Definition 1 makes sense if, say, $U$ belongs to $L^{2}(0, T)$. Closing the loop, a hidden regularity property of the solutions is needed to ensure all terms are defined 1

\footnotetext{
${ }^{1}$ In the language of systems theory, one may say that the Neuman input operator is unbounded with respect to the state space $\mathcal{X}$.
}

Assumption 2. The function $g: \mathbb{R} \rightarrow \mathbb{R}$ is continuous and nondecreasing. ${ }^{2}$

We show that the closed-loop system generates a dynamical system on $\mathcal{X}$ by defining the operators $\mathcal{S}_{t}$ for $t \geq 0$ as follows:

$$
\forall \mathbf{X}=\left[\mathbf{u}^{0}, \mathbf{u}^{1}\right] \in \mathcal{X}, \quad \mathcal{S}_{t}(\mathbf{X}) \triangleq\left[\mathbf{u}(t), \mathbf{u}^{\prime}(t)\right] \in \mathcal{X},
$$

where $\mathbf{u}$ is the unique solution associated with initial data $\left[\mathbf{u}^{0}, \mathbf{u}^{1}\right]$.

Theorem 1 (Hadamard well-posedness). Let $\left[\mathbf{u}^{0}, \mathbf{u}^{1}\right] \in \mathcal{X}$. Under Assumptions 1 and 2, there exists a unique (weak) solution $\mathbf{u} \in \mathcal{C}\left(\mathbb{R}^{+}, V\right) \cap \mathcal{C}^{1}\left(\mathbb{R}^{+}, H\right)$ to (1) with feedback (2) and initial data $\left[\mathbf{u}^{0}, \mathbf{u}^{1}\right]$. The solution $\mathbf{u}$ enjoys the following hidden regularity property: for all $T>0$,

$$
u(L, \cdot) \in H^{1}(0, T) \text { and } g\left(\partial_{t} u(L, \cdot)\right) \in L^{2}(0, T) .
$$

Moreover, we can associate with (1) and control law (2) the semigroup $\mathfrak{S}=\left\{\mathcal{S}_{t}\right\}_{t \geq 0}$ of nonlinear continuous operators on $\mathcal{X}$ as defined in 9 .

Theorem 1 is proved in Section V. In the following proposition, we give some additional regularity when the initial datum is smooth and verifies a compatibility condition. We write $W \triangleq\left[H^{2}(\Omega) \times \mathbb{R}\right] \cap V$, equipped with the scalar product inherited from $H^{2}(\Omega) \times \mathbb{R}$.

Proposition 1 (Strong solutions). Let $T>0$. If $\left[\mathbf{u}^{0}, \mathbf{u}^{1}\right]$ belongs to $W \times V$ and verifies $\partial_{x} u^{0}(L)=-g\left(u^{1}(L)\right)$, then,

$$
\mathbf{u} \in L^{\infty}(0, T ; W), \quad \mathbf{u}^{\prime} \in L^{\infty}(0, T ; V) .
$$

Moreover, any weak solution $\mathbf{u}$ is the limit of a sequence of strong solutions $\left[\mathbf{u}_{n}, \mathbf{u}_{n}^{\prime}\right]$ in $\mathcal{C}([0, T], \mathcal{X})$, and $\partial_{t} u_{n}(L, \cdot) \rightarrow$ $\partial_{t} u(L, \cdot)$ in $L^{2}(0, T)$.

Proposition 1 is a byproduct of the proof of Theorem 1 and is used to justify computations performed in Section III.

\section{StABILITY ANALYSIS OF THE CLOSED-LOOP SYSTEM}

Next, we analyze the stability of the closed-loop system when the feedback is linear. We introduce the energy functional $\mathcal{E}$ defined on $\mathcal{X}$ by

$$
\mathcal{E}(\mathbf{u}, \mathbf{v}) \triangleq \frac{1}{2}\left[\|\mathbf{v}\|_{H}^{2}+a(\mathbf{u}, \mathbf{u})\right]
$$

which is, in the context of abstract wave equations, the natural "mechanical" energy. Here, for all $\mathbf{u}$ in $V$ and $\mathbf{v}=(v, \gamma)$ in $H$,

$$
\mathcal{E}(\mathbf{u}, \mathbf{v})=\frac{1}{2} \int_{\Omega}|v(x)|^{2}+\left|\partial_{x} u(x)\right|^{2} \mathrm{~d} x+\frac{1}{2}|\gamma|^{2} .
$$

For any $\rho$ and $\mu$ in $H^{1}(\Omega)$, we define a weighted energy functional (or Lyapunov function candidate):

$$
\Gamma_{\rho, \mu}(\mathbf{u}, \mathbf{v}) \triangleq \frac{1}{2} \int_{\Omega}\left[\begin{array}{c}
v \\
\partial_{x} u
\end{array}\right]^{\top}\left[\begin{array}{cc}
\mu & \rho \\
\rho & \mu
\end{array}\right]\left[\begin{array}{c}
v \\
\partial_{x} u
\end{array}\right]+\frac{1}{2} \mu(0)|\gamma|^{2} .
$$

\footnotetext{
${ }^{2}$ It is a specificity of the one-dimensional case that no additional growth assumption on $g$ is required to obtain weak solutions for such feedback.
} 
It follows from the boundedness of the weights $\rho$ and $\mu$ that there exists a constant $M_{\rho, \mu}>0$ such that

$$
\forall[\mathbf{u}, \mathbf{v}] \in \mathcal{X}, \quad \Gamma_{\rho, \mu}(\mathbf{u}, \mathbf{v}) \leq M_{\rho, \mu} \mathcal{E}(\mathbf{u}, \mathbf{v})
$$

A sufficient condition for the converse inequality to hold is the existence of $C_{1}, C_{2}>0$ such that

$$
\mu(x) \geq C_{1} \text { and } \mu(x)^{2}-\rho(x)^{2} \geq C_{2}
$$

holds for all $x$ in $\Omega$. We also remark that $\mathcal{E}$ and $\Gamma$ are continuous on $\mathcal{X}$. We note that the position does not appear in the energy functionals and without Poincaré-type inequalities, one cannot directly infer an estimate of the norm of the position. Stabilization shall be investigated with respect to the following invariant set, which is the line composed of constant solutions:

$$
\mathfrak{A} \triangleq\{[\theta \mathbb{1}, 0] \in \mathcal{X}: \theta \in \mathbb{R}\}
$$

where $\mathbb{1} \triangleq((x \mapsto 1), 1)$. Observe that $\mathfrak{A}$ is exactly the subset of $\mathcal{X}$ where the mechanical energy $\mathcal{E}$ vanishes. For any $[\mathbf{u}, \mathbf{v}]$ in $\mathcal{X}$,

$$
\operatorname{dist}([\mathbf{u}, \mathbf{v}], \mathfrak{A})^{2} \leq C \mathcal{E}(\mathbf{u}, \mathbf{v})
$$

where $C$ is some positive constant. This is a consequence of the Poincaré-Wirtinger inequality - see [2] for instance. From now on, we are interested in stabilizing system (1) using a proportional feedback, i.e.

$$
U(t)=-\alpha \partial_{t} u(L, t)
$$

where $\alpha$ is a positive gain to be tuned.

Assumption 3. The function $F$ is $q$-Lipschitz for some $q<$ 1/2. Also, $F(0)=0$.

The condition $F(0)=0$ is quite natural when dealing with friction. Assumption 3 is meant to be in force in the context of nonlinear anti-damping ${ }^{3}$

Theorem 2 (Exponential stability). Under Assumption 3 and with $\alpha=1, \mathfrak{A}$ uniformly and exponentially attracts the bounded sets of $\mathcal{X}$. More precisely, there exist two positive constants $M$ and $\eta$ such that for all solutions $\mathbf{u}$ to (1),

$$
\mathcal{E}\left(\mathbf{u}(t), \mathbf{u}^{\prime}(t)\right) \leq M \mathcal{E}\left(\mathbf{u}^{0}, \mathbf{u}^{1}\right) \exp (-\eta t)
$$

Moreover, for each solution $\mathbf{u}, \mathbf{u}(t)$ converges in $V$ to some constant function $\mathbf{u}_{\infty}$ when $t \rightarrow+\infty$.

Proof. We pick a solution $\mathbf{u}$ and, for the sake of concision, we denote by $\Gamma_{\rho, \mu}^{\mathbf{u}}$ the function $t \in \mathbb{R}^{+} \mapsto \Gamma_{\rho, \mu}\left(\mathbf{u}(t), \mathbf{u}^{\prime}(t)\right)$, which is continuous. Take $\rho, \mu \in H^{1}(\Omega)$. Assume temporarily that $\mathbf{u}$ is a strong solution. For all $\tau \geq 0$, denoting by

\footnotetext{
${ }^{3}$ Alternatively, if $F$ has a stabilizing effect, e.g. $F(0)=0, F$ nonincreasing, it can be relaxed to $F$ locally Lipschitz - with appropriate modifications in the proof of well-posedness.
}

$Q_{\tau}$ the rectangle $\Omega \times(0, \tau)$, we have the following identity:

$$
\begin{array}{r}
\left.\Gamma_{\rho, \mu}^{\mathbf{u}}(t)\right|_{0} ^{\tau}=-\frac{1}{2} \iint_{Q_{\tau}}\left[\begin{array}{l}
\partial_{t} u \\
\partial_{x} u
\end{array}\right]^{\top}\left[\begin{array}{ll}
\rho^{\prime} & \mu^{\prime} \\
\mu^{\prime} & \rho^{\prime}
\end{array}\right]\left[\begin{array}{l}
\partial_{t} u \\
\partial_{x} u
\end{array}\right] \\
+\frac{1}{2}\left[\rho(L)\left(1+\alpha^{2}\right)-2 \alpha \mu(L)\right] \int_{0}^{\tau}\left|\partial_{t} u(L, t)\right|^{2} \mathrm{~d} t \\
-\frac{\rho(0)}{2} \int_{0}^{\tau}\left|\partial_{t} u(0, t)\right|^{2}+\left|\partial_{x} u(0, t)\right|^{2} \mathrm{~d} t \\
+\mu(0) \int_{0}^{\tau} F\left(\partial_{t} u(0, t)\right) \partial_{t} u(0, t) \mathrm{d} t
\end{array}
$$

Equation 21 is obtained by multiplying the wave equation $\partial_{t t} u(x, t)-\partial_{x x} u(x, t)=0$ by $\phi(x, y)=\mu(x) \partial_{t} u(x, t)+$ $\rho(x) \partial_{x} u(x, t) \in H^{1}\left(Q_{\tau}\right)$, integrating over $Q_{\tau}$, and performing a few integrations by parts. If $\rho$ and $\mu$ are nonnegative, writing $|F(s)| \leq q|s|$ where $q$ is the Lipschitz constant in Assumption 3, we have the following inequality:

$$
\begin{array}{r}
\left.\Gamma_{\rho, \mu}^{\mathbf{u}}(t)\right|_{0} ^{\tau} \leq-\frac{1}{2} \iint_{Q_{\tau}}\left[\begin{array}{l}
\partial_{t} u \\
\partial_{x} u
\end{array}\right]^{\top}\left[\begin{array}{ll}
\rho^{\prime} & \mu^{\prime} \\
\mu^{\prime} & \rho^{\prime}
\end{array}\right]\left[\begin{array}{l}
\partial_{t} u \\
\partial_{x} u
\end{array}\right] \\
+\frac{1}{2}\left[\rho(L)\left(1+\alpha^{2}\right)-2 \alpha \mu(L)\right] \int_{0}^{\tau}\left|\partial_{t} u(L, t)\right|^{2} \mathrm{~d} t \\
+\frac{1}{2}[2 q \mu(0)-\rho(0)] \int_{0}^{\tau}\left|\partial_{t} u(0, t)\right|^{2} \mathrm{~d} t .
\end{array}
$$

Take $\mu(x)=1$. For $[16$ to hold, it suffices to have $\rho(x) \leq$ $1-\epsilon$ for some $\epsilon>0$. Now, we derive some sufficient conditions for the energy to decay exponentially. It suffices to have $\rho(0) \geq 2 q+\epsilon$, and $\rho^{\prime}(x) \geq \epsilon$ a.e. as well as

$$
\rho(L) \leq \frac{2 \alpha}{1+\alpha^{2}}-\epsilon
$$

for some $\epsilon>0$. Since $q<1 / 2$, there exists an increasing affine function $\rho$ such that $\rho(0)>2 q$ and $\rho(L)<1$. Let $\alpha=1$ so that 23 holds. As a result, by Grönwall's lemma, we obtain the following: with this particular choice of $\rho$ and $\mu$, there exists a positive constant (solution independent) $\eta$ such that

$$
\forall t \geq 0, \quad \Gamma_{\rho, \mu}^{\mathbf{u}}(t) \leq \Gamma_{\rho, \mu}^{\mathbf{u}}(0) \exp (-\eta t)
$$

By a density-continuity argument, the uniform estimate 24 holds for weak solutions as well - see Proposition 11 Since (16) holds, then there exists $M>0$ such that (20) is verified by any solution. As for the second statement of Theorem 2, let $\mathbf{u}$ be a solution. Take an increasing sequence of nonnegative real numbers $t_{n}$ such that $t_{n} \rightarrow+\infty$ when $n \rightarrow+\infty$. Then $\left\{\mathbf{u}\left(t_{n}\right)\right\}_{n \geq 0}$ is a Cauchy sequence in $V$. Indeed, for any $m \geq n$, writing the variation of $\mathbf{u}(t)$ between $t_{n}$ and $t_{m}$, we have

$$
\begin{aligned}
\left\|\mathbf{u}\left(t_{m}\right)-\mathbf{u}\left(t_{n}\right)\right\|_{H} & \leq \int_{t_{n}}^{t_{m}}\left\|\mathbf{u}^{\prime}(s)\right\|_{H} \mathrm{~d} s \\
& \leq M^{\prime} \int_{t_{n}}^{+\infty} \exp (-\eta s / 2) \mathrm{d} s
\end{aligned}
$$

which converges to 0 when $n \rightarrow+\infty$; also, we already know that $a(\mathbf{u}(t), \mathbf{u}(t)) \rightarrow 0$ when $t \rightarrow+\infty$, thus $\| \mathbf{u}\left(t_{m}\right)-$ $\mathbf{u}\left(t_{n}\right) \|_{V}$ can be arbitrarily small. Using a similar argument, 
one verifies that the limit does not depend on the sequence $\left\{t_{n}\right\}$. Therefore, $\mathbf{u}(t)$ converges to some $\mathbf{u}_{\infty} \in V$ and $\mathbf{u}_{\infty}$ is constant by $(18)$.

\section{NUMERICAL SIMULATIONS}

We provide some numerical computations for illustrative purposes.

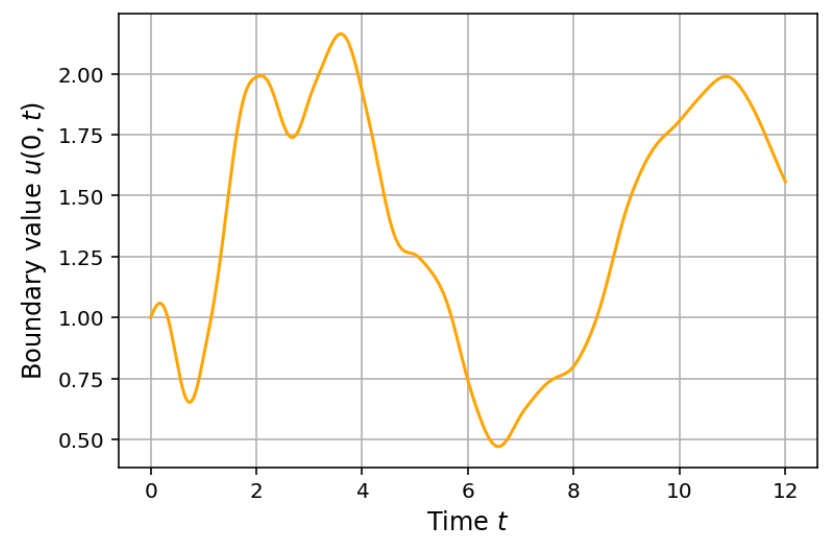

Fig. 1. Evolution of the boundary position $u(0, t)$ over time. It obeys a second-order differential equation coupled with the wave equation.

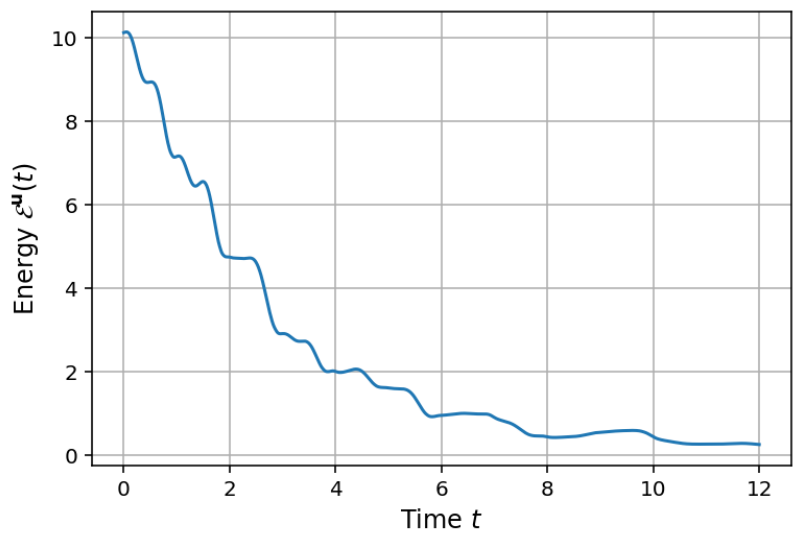

Fig. 2. Evolution of the mechanical energy $\mathcal{E}\left(\mathbf{u}(t), \mathbf{u}^{\prime}(t)\right)$ over time. The uncontrolled system would be unstable due to the boundary anti-damping.

We discretize (1) using finite elements over space and finite differences over time, on the basis of the functional formulation of the problem. Figures 1 and 2 are obtained with the following parameters: we take $\alpha=1, F(x)=q x$ with $q=0.1$ and $L=1$. The domain $\Omega$ is discretized into 100 points and the time step is set to 0.001 . Further computations suggest that the condition on $q$ derived in Theorem 2 is nearly sharp as taking $q=0.5$ leads to an exponentially unstable system. Computations also suggest that the proportional feedback is not robust to (numerical) errors: taking values of $q$ slightly below 0.5 induces unclear situations where an exponential decay is not easily identifiable.

\section{PRoOF of THE HADAMARd THEOREM}

The proof of Theorem 1 relies on nonlinear semigroup techniques and appropriate energy estimates. We begin this section with some remarks on the functional settings of the problem. The following result states some useful properties of the spaces $H$ and $V$.

Lemma 1. $V$ is a separable Hilbert space isomorphic to $H^{1}(\Omega)$. Moreover, $V$ is a dense subset of $H$.

Since $\|\mathbf{u}\|_{V}^{2}=\|\mathbf{u}\|_{H}^{2}+a(\mathbf{u}, \mathbf{u})$, we see that $V$ is continuously embedded into $H$. We denote $H^{\prime}$ the topological dual of $H$. By Riesz representation theorem, we make the identification $H \simeq H^{\prime}$. Because $V$ is a dense subset of $H$, the latter can be identified as a dense subset of $V^{\prime}$, leading to the classical injection chain $V \hookrightarrow H \simeq H^{\prime} \hookrightarrow V^{\prime}$, where each space is dense and continuously embedded into the following one. We denote by $A$ the continuous operator from $V$ into $V^{\prime}$ defined by $\left\langle A \mathbf{u}_{1}, \mathbf{u}_{2}\right\rangle_{V} \triangleq a\left(\mathbf{u}_{1}, \mathbf{u}_{2}\right)$ for all $\mathbf{u}_{1}, \mathbf{u}_{2}$ in $V$. Next, define an unbounded (nonlinear) operator $\mathcal{A}_{g}$ on $\mathcal{X}$ by

$$
\begin{aligned}
& \mathcal{D}\left(\mathcal{A}_{g}\right) \triangleq\left\{[\mathbf{u}, \mathbf{v}] \in W \times V: \partial_{x} u(L)=-g(v(L))\right\} \\
& \forall \mathbf{X}=[\mathbf{u}, \mathbf{v}] \in \mathcal{D}\left(\mathcal{A}_{g}\right), \quad \mathcal{A}_{g}(\mathbf{X}) \triangleq-\left[\begin{array}{c}
\mathbf{v} \\
\left(\partial_{x x} u, \partial_{x} u(0)\right)
\end{array}\right] .
\end{aligned}
$$

Note that the domain $\mathcal{D}\left(\mathcal{A}_{g}\right)$ need not be a subspace. We start with the following first-order abstract Cauchy problem:

$$
\left\{\begin{array}{l}
\dot{\mathbf{X}}(t)+\mathcal{A}_{g}(\mathbf{X}(t))=\mathcal{F}(\mathbf{X}(t)) \\
\mathbf{X}(0)=\mathbf{X}^{0},
\end{array}\right.
$$

where $\mathcal{F}$ is the nonlinear perturbation operator on $\mathcal{X}$ defined by $\mathcal{F}(\mathbf{X}) \triangleq[0, B(\mathbf{v})]$ for all $\mathbf{X}=[\mathbf{u}, \mathbf{v}] \in \mathcal{X}$. We see that $\mathcal{F}$ is Lipschitz, since the $H$-valued mapping $B$ is Lipschitz. We wish to prove that 27) is a Lipschitz perturbation of an evolution equation with maximal monotone generator, hence the following result.

Proposition 2. The unbounded operator $\mathcal{A}_{g}+$ id is maximal monotone.

Proof. We start with the monotonicity, and then we shall prove the surjectivity of $\left[\mathcal{A}_{g}+\mathrm{id}\right]+\mathrm{id}$.

Step 1: Monotonicity. Let $\mathbf{X}_{1}=\left[\mathbf{u}_{1}, \mathbf{v}_{1}\right]$ and $\mathbf{X}_{2}=$ $\left[\mathbf{u}_{2}, \mathbf{u}_{2}\right]$ in $\mathcal{D}\left(\mathcal{A}_{g}\right)$. We denote $\mathbf{u}_{1}-\mathbf{u}_{2}$ (resp. $\left.\mathbf{v}_{1}-\mathbf{v}_{2}\right)$ by $\mathbf{u}$ (resp. v), and also $\mathbf{X}_{1}-\mathbf{X}_{2}$ by $\mathbf{X}$. We have

$$
\begin{aligned}
\left(\mathcal{A}_{g}\left(\mathbf{X}_{1}\right)-\mathcal{A}_{g}\left(\mathbf{X}_{2}\right), \mathbf{X}\right)_{\mathcal{X}}=-(\mathbf{u}, \mathbf{v})_{V} \\
-\left(\partial_{x x} u, v\right)_{L^{2}(\Omega)}-\partial_{x} u(0) v(0)
\end{aligned}
$$

Recall that $(\mathbf{u}, \mathbf{v})_{V}=(\mathbf{u}, \mathbf{v})_{H}+a(\mathbf{u}, \mathbf{v})$. Moreover, by integration by parts, we have $a(\mathbf{u}, \mathbf{v})=-\left(\partial_{x x} u, v\right)_{L^{2}(\Omega)}+$ $\partial_{x} u(L) v(L)-\partial_{x} u(0) v(0)$. Thus, from 28) we obtain

$$
\left(\mathcal{A}_{g}\left(\mathbf{X}_{1}\right)-\mathcal{A}_{g}\left(\mathbf{X}_{2}\right), \mathbf{X}\right)_{\mathcal{X}}=-(\mathbf{u}, \mathbf{v})_{H}-\partial_{x} u(L) v(L) .
$$

By definition of $\mathcal{D}\left(\mathcal{A}_{g}\right)$ and Assumption 2, $-\partial_{x} u(L) v(L)=$ $\left[g\left(v_{1}(L)\right)-g\left(v_{2}(L)\right)\right] v(L) \geq 0$, hence

$$
\left(\mathcal{A}_{g}\left(\mathbf{X}_{1}\right)-\mathcal{A}_{g}\left(\mathbf{X}_{2}\right), \mathbf{X}\right)_{\mathcal{X}} \geq-(\mathbf{u}, \mathbf{v})_{H}
$$

From 29 we then obtain

$$
\begin{aligned}
\left(\mathcal{A}_{g}\left(\mathbf{X}_{1}\right)-\right. & \left.\mathcal{A}_{g}\left(\mathbf{X}_{2}\right)+\mathbf{X}, \mathbf{X}\right)_{\mathcal{X}} \\
& \geq-(\mathbf{u}, \mathbf{v})_{H}+\|\mathbf{u}\|_{V}^{2}+\|\mathbf{v}\|_{H}^{2} \\
& \geq-(\mathbf{u}, \mathbf{v})_{H}+\frac{1}{2}\|\mathbf{u}\|_{H}^{2}+\frac{1}{2}\|\mathbf{v}\|_{H}^{2} \geq 0
\end{aligned}
$$


which is the desired result.

Step 2: Surjectivity. Take $\mathbf{Y}=\left[\mathbf{f}_{1}, \mathbf{f}_{2}\right] \in \mathcal{X}$. Let us prove that there exists $\mathbf{X}=[\mathbf{u}, \mathbf{v}] \in \mathcal{D}\left(\mathcal{A}_{g}\right)$ such that $\mathcal{A}_{g}(\mathbf{X})+$ $2 \mathbf{X}=\mathbf{Y}$, i.e.

$$
\begin{cases}\left(-\partial_{x x} u,-\partial_{x} u(0)\right)+2 \mathbf{v}=\mathbf{f}_{2} & \text { in } H \\ 2 \mathbf{u}-\mathbf{v}=\mathbf{f}_{1} & \text { in } V .\end{cases}
$$

Replacing $\mathbf{u}$ with $\left(\mathbf{f}_{1}+\mathbf{v}\right) / 2$ in 32 and using the condition $\partial_{x} u(L)=-g(v(L))$, we may start by finding $\mathbf{v} \in V$ such that for all $\mathbf{w} \in V$,

$$
\begin{aligned}
\frac{1}{2} a(\mathbf{v}, \mathbf{w})+\frac{1}{2} g(v(L)) & w(L)+2(\mathbf{v}, \mathbf{w})_{H} \\
= & -\frac{1}{2} a\left(\mathbf{f}_{1}, \mathbf{w}\right)+\left(\mathbf{f}_{2}, \mathbf{w}\right)_{H} .
\end{aligned}
$$

Define $\Theta: V \rightarrow V^{\prime}$ by, for all $\mathbf{v}, \mathbf{w} \in V$,

$$
\langle\Theta(\mathbf{v}), \mathbf{w}\rangle_{V} \triangleq a(\mathbf{v}, \mathbf{w})+g(v(L)) w(L)+4(\mathbf{v}, \mathbf{w})_{H},
$$

and also $L \in V^{\prime}$ by $\langle L, \mathbf{w}\rangle_{V} \triangleq a\left(\mathbf{f}_{1}, \mathbf{w}\right)+2\left(\mathbf{f}_{2}, \mathbf{w}\right)_{H}$. Then, reformulating (33), we seek $\mathbf{v} \in V$ such that

$$
\Theta(\mathbf{v})=L \text { in } V^{\prime} .
$$

We wish to apply Lemma 3 given in Appendix to $\Theta$. As a Hilbert space, $V$ is reflexive; it is also separable - see Lemma 1. For all $\mathbf{w}_{1}, \mathbf{w}_{2} \in V,\left\langle\Theta\left(\mathbf{w}_{1}\right)-\right.$ $\left.\Theta\left(\mathbf{w}_{2}\right), \mathbf{w}_{1}-\mathbf{w}_{2}\right\rangle_{V}=a\left(\mathbf{w}_{1}-\mathbf{w}_{2}, \mathbf{w}_{1}-\mathbf{w}_{2}\right)+\left[g\left(w_{1}(L)\right)-\right.$ $\left.g\left(w_{2}(L)\right)\right]\left(w_{1}(L)-w_{2}(L)\right)+4\left\|\mathbf{w}_{1}-\mathbf{w}_{2}\right\|_{H}^{2} \geq 0$, which proves the monotonicity. Items (2) and (3) are easily verified using continuity arguments. Finally, let $\mathbf{w} \in V$; we have $\langle L, \mathbf{w}\rangle_{V^{\prime}, V} \leq\|L\|_{V^{\prime}}\|\mathbf{w}\|_{V}$ and $\langle\Theta(\mathbf{w}), \mathbf{w}\rangle_{V^{\prime}, V} \geq\|\mathbf{w}\|_{V}^{2}$, so that $\langle\Theta(\mathbf{w}), \mathbf{w}\rangle_{V^{\prime}, V}-\langle L, \mathbf{w}\rangle_{V^{\prime}, V} \rightarrow+\infty$ when $\|\mathbf{w}\|_{V} \rightarrow$ $+\infty$, which implies the desired property. We deduce from Lemma 3 that there exists $\mathbf{v} \in V$ such that 35 holds. Then, let $\mathbf{u} \triangleq\left(\mathbf{f}_{1}+\mathbf{v}\right) / 2 \in V$. For all $\mathbf{w} \in V$, we have

$$
a(\mathbf{u}, \mathbf{w})+g(v(L)) w(L)+2(\mathbf{v}, \mathbf{w})_{H}=\left(\mathbf{f}_{2}, \mathbf{w}\right)_{H} .
$$

Since $f_{2}$ belongs to $L^{2}(\Omega)$, taking $\phi=(\phi, 0)$ in (36), where $\phi$ is an arbitrary element of $\mathcal{C}_{c}^{\infty}(\Omega)$, allows us to prove that $u \in H^{2}(\Omega)$. Now, integrating by parts in (36) gives

$$
\begin{gathered}
\partial_{x} u(L) w(L)-\partial_{x} u(0) w(0)+g(v(L)) w(L)+2 v(0) w(0) \\
=f_{2}(0) w(0),
\end{gathered}
$$

holding for all $\mathbf{w} \in V$. Pick a triangular function $\rho$ such that $\rho(0)=1$ and $\rho(L)=0$. Then $(\rho, 1) \in V$. Evaluating (37) with $\rho(x)$ and then $\rho(L-x)$ yields $-\partial_{x} u(0)+2 v(0)=f_{2}(0)$ and $\partial_{x} u(L)=-g(v(L))$, hence $\mathbf{X}=(\mathbf{u}, \mathbf{v}) \in \mathcal{D}\left(\mathcal{A}_{g}\right)$ and $\mathcal{A}_{g}(\mathbf{X})+2 \mathbf{X}=\mathbf{Y}$, which concludes the proof.

In order to consider any initial datum in the state space $\mathcal{X}$, we complete Proposition 2 with the following lemma.

Lemma 2. $\mathcal{D}\left(\mathcal{A}_{g}\right)$ is dense in $\mathcal{X}$.

We can finally prove the Hadamard theorem. Considering the evolution equation 27 with a sequence of approximate initial data in $\mathcal{D}\left(\mathcal{A}_{g}\right)$ provides strong solutions that converge in $\mathcal{C}([0, T], \mathcal{X})$. Then, one has to prove that the limit is a weak solution. For that purpose, using a multiplier method, we retrieve a standard hidden regularity property of the wave equation.

Proof of Theorem 1. We split the proof into four steps.

Step 1: Approximate solutions. Since $\mathcal{D}\left(\mathcal{A}_{g}\right)$ is dense in $\mathcal{X}$, we can pick a sequence of vectors $\mathbf{X}_{n}^{0}=\left[\mathbf{u}_{n}^{0}, \mathbf{u}_{n}^{1}\right] \in$ $\mathcal{D}\left(\mathcal{A}_{g}\right)$ that converges to $\left[\mathbf{u}^{0}, \mathbf{u}^{1}\right]$ in $\mathcal{X}$. For each $n \geq 0$, there exists a (unique) strong solution $\mathbf{X}_{n} \in W^{1, \infty}\left(\mathbb{R}^{+}, \mathcal{X}\right)$ to the following reformulation of 27):

$$
\left\{\begin{array}{l}
\dot{\mathbf{X}}(t)+\mathcal{A}_{g}(\mathbf{X}(t))+\mathbf{X}(t)=\mathcal{F}(\mathbf{X}(t))+\mathbf{X}(t) \\
\mathbf{X}(0)=\mathbf{X}_{n}^{0} .
\end{array}\right.
$$

Indeed, Proposition 2 states that $\mathcal{A}_{g}+$ id is maximal monotone, and $\mathcal{F}+$ id is still Lipschitz. Existence is given by [11, Corollary 4.1]. For all $n \geq 0, \mathbf{X}_{n}=\left[\mathbf{u}_{n}, \mathbf{u}_{n}^{\prime}\right]$ verifies ${ }^{4}$

$$
\mathbf{u}_{n}^{\prime \prime}(t)-\left(\partial_{x x} u_{n}(t), \partial_{x} u_{n}(0, t)\right)=B\left(\mathbf{u}_{n}^{\prime}(t)\right) \quad \text { in } H,
$$

in the sense of strong differentiation, for a.e. $t>0$, with $\mathbf{X}_{n}$ taking values in $\mathcal{D}\left(\mathcal{A}_{g}\right)$. We pick $\mathbf{w}$ in $V$ and take the scalar product of (40) with $\mathbf{w}$. With an integration by parts, and using the definition of $\mathcal{D}\left(\mathcal{A}_{g}\right)$, we obtain

$$
\begin{array}{r}
\left(\mathbf{u}_{n}^{\prime \prime}(t), \mathbf{w}\right)_{H}+a\left(\mathbf{u}_{n}(t), \mathbf{w}\right)=\left(B\left(\mathbf{u}_{n}^{\prime}(t)\right), \mathbf{w}\right)_{H} \\
-g\left(\partial_{t} u_{n}(L, t)\right) w(L)
\end{array}
$$

for a.e. $t>0$, which means that, $\mathbf{w}$ being arbitrary,

$$
\mathbf{u}_{n}^{\prime \prime}(t)+A \mathbf{u}_{n}(t)=B\left(\mathbf{u}_{n}^{\prime}(t)\right)-g\left(\partial_{t} u_{n}(L, t)\right) \delta_{L} \quad \text { in } V^{\prime}
$$

for a.e. $t>0$. Let $T>0$. Since $\mathbf{u}_{n} \in L^{\infty}(0, T ; V) \cap$ $W^{1, \infty}(0, T ; H)$ and $\mathbf{u}_{n}^{\prime} \in W^{1,2}\left(0, T ; V^{\prime}\right)$, a standard timeregularization argument - omitted here - allows us to infer from 41 that the distributional identity (8) holds for all $\phi \in$ $\mathcal{C}([0, T], V) \cap \mathcal{C}^{1}([0, T], H)$, meaning that $\mathbf{u}_{n}$ is indeed a weak solution to (1) in the sense of Definition 1] with initial data $\left[\mathbf{u}_{n}^{0}, \mathbf{v}_{n}^{0}\right]$. Besides, as a consequence of [11, Corollary 4.1], $\mathbf{X}_{n}$ takes values in $\mathcal{D}\left(\mathcal{A}_{g}\right)$ and $\left\|\mathcal{A}_{g}\left(\mathbf{X}_{n}\right)\right\|_{\mathcal{X}}$ is bounded on $[0, T]$, meaning that

$$
\mathbf{u}_{n} \in L^{\infty}(0, T ; W), \quad \mathbf{u}_{n}^{\prime} \in L^{\infty}(0, T ; V) ;
$$

also, $\partial_{t} u_{n}(0, \cdot)$ is absolutely continuous and $\partial_{t t} u_{n}(0, t)-$ $\partial_{x} u_{n}(0, t)=F\left(\partial_{t} u_{n}(0, t)\right)$ Thus, $u_{n}(0, \cdot)$ belongs to $H^{2}(0, T)$ with the desired weak derivatives. It can be inferred from the distributional identity (8) and the additional regularity given by (42) that $u_{n}(x, t)$ belongs to $H^{2}\left(Q_{T}\right)$ and verifies the wave equation on $Q_{T}$.

Step 2: Uniform convergence. The convergence of the sequence $\left\{\mathbf{X}_{n}\right\}$ in $\mathcal{C}([0, T], \mathcal{X})$ for any $T>0$ follows from the standard monotonicity argument. We denote its limit by $\mathbf{X}=\left[\mathbf{u}, \mathbf{u}^{\prime}\right]$. Coming back to the sequence of approximate strong solutions, for all $(m, n)$ in $\mathbb{N}^{2}$, we write $\mathbf{w}_{m n} \triangleq \mathbf{u}_{m}-$ $\mathbf{u}_{n}$. We also write $\mathbf{w}_{m n}^{0} \triangleq \mathbf{w}_{m n}(0)$ and $\mathbf{w}_{m n}^{1} \triangleq \mathbf{w}_{m n}^{\prime}(0)$. Multiplying the wave equation by $x \partial_{x} w_{m n}(x, t) \in H^{1}\left(Q_{\tau}\right)$,

\footnotetext{
${ }^{4}$ One should verify that the second coordinate is indeed the weak derivative of the first coordinate.
} 
we obtain, after several integrations by parts, the following boundary estimate:

$$
\begin{aligned}
\int_{0}^{\tau} \mid g\left(\partial_{t} u_{m}(L, t)\right) & -\left.g\left(\partial_{t} u_{n}(L, t)\right)\right|^{2}+\left|\partial_{t} w_{m n}(L, t)\right|^{2} \mathrm{~d} t \\
= & \left.\frac{2}{L} \int_{\Omega} x \partial_{t} w_{m n}(x, t) \partial_{x} w_{m n}(x, t) \mathrm{d} x\right|_{0} ^{\tau} \\
& +\frac{1}{L} \iint_{Q_{\tau}}\left|\partial_{t} w_{m n}\right|^{2}+\left|\partial_{x} w_{m n}\right|^{2}
\end{aligned}
$$

From 43, using Cauchy-Schwarz and Young inequalities, and the Lipschitz property of $B$, we obtain the following:

$$
\begin{aligned}
& \int_{0}^{\tau} \mid g\left(\partial_{t} u_{m}(L, t)\right)-\left.g\left(\partial_{t} u_{n}(L, t)\right)\right|^{2}+\left|\partial_{t} w_{m n}(L, t)\right|^{2} \mathrm{~d} t \\
& \leq M \sup _{t \in[0, \tau]}\left[\left\|\mathbf{w}_{m n}\right\|_{V}^{2}+\left\|\mathbf{w}_{m n}\right\|_{H}^{2}\right]
\end{aligned}
$$

for all $\tau \in[0, T]$ and some $M>0$. As a consequence, $\left\{\partial_{t} u_{n}(L, \cdot)\right\}$ and $\left\{g\left(\partial_{t} u_{n}(L, \cdot)\right)\right\}$ are Cauchy sequences in $L^{2}(0, T)$. Since $u_{n}(L, \cdot)$ converges (in particular) in $L^{2}(0, T)$, it follows that $\left\{u_{n}(L, \cdot)\right\}$ is a Cauchy sequence in $H^{1}(0, T)$, meaning that $u(L, \cdot)$ belongs in fact to $H^{1}(0, T)$, which is the desired hidden regularity property. By continuity of $g$ and unicity of the limit, $g\left(\partial_{t} u_{n}(L, \cdot)\right)$ converges to $g\left(\partial_{t} u(L, \cdot)\right)$ in $L^{2}(0, T)$ - one has to consider a subsequence converging for a.e. $t \in(0, T)$.

Step 3: Existence of weak solutions. Each $\mathbf{u}_{n}$ verifies the distributional identity (8) for all test-functions $\phi$ in $\mathcal{C}_{c}(0, T ; V) \cap \mathcal{C}_{c}^{1}(0, T ; H)$. We pick $\phi$ and we let $n \rightarrow+\infty$. As $\left[\mathbf{u}_{n}, \mathbf{u}_{n}^{\prime}\right]$ converges to $\left[\mathbf{u}, \mathbf{u}^{\prime}\right]$ in $\mathcal{C}([0, T], \mathcal{X}), \partial_{t} u_{n}(L, \cdot)$ converges to $\partial_{t} u(L, \cdot)$ in $L^{2}(0, T)$, and $g\left(\partial_{t} u_{n}(L, \cdot)\right)$ converges to $g\left(\partial_{t} u(L, \cdot)\right)$ in $L^{2}(0, T)$, we obtain that $\mathbf{u}$ verifies (8) as well and is therefore a weak solution to (1).

Step 4: Unicity and Hadamard continuity. Let $w \triangleq$ $\mathbf{u}_{1}-\mathbf{u}_{2}$, where $\mathbf{u}_{1}$ and $\mathbf{u}_{2}$ are two arbitrary weak solutions. The following energy identity holds:

$$
\begin{array}{r}
\left.\mathcal{E}^{\mathbf{w}}(t)\right|_{0} ^{\tau}=\int_{0}^{\tau}\left(B\left(\mathbf{u}_{1}^{\prime}(t)\right)-B\left(\mathbf{u}_{2}^{\prime}(t)\right), \mathbf{w}^{\prime}(t)\right)_{H} \\
-\left[g\left(\partial_{t} u_{1}(L, t)\right)-g\left(\partial_{t} u_{2}(L, t)\right)\right] \partial_{t} w(L, t) \mathrm{d} t
\end{array}
$$

for any $0 \leq \tau$. It is obtained by multipling the wave equation by $\partial_{t} w(x, t)$ and integrating over $Q_{\tau}$. From there, using the fact that $g$ is nondecreasing and $B$ is Lipschitz, since we also have $\left.\|\mathbf{w}(t)\|_{H}^{2}\right|_{0} ^{\tau} \leq 2 \int_{0}^{\tau}\|\mathbf{w}\|_{H}\left\|\mathbf{w}^{\prime}\right\|_{H}$, we can apply the standard Grönwall argument to obtain unicity and continuity with respect to initial conditions, proving that the operators $\mathcal{S}_{t}$ introduced in $(9)$ are well-defined and continuous. At this point, Proposition 1 is also proved.

\section{CONCLUSION AND PERSPECTIVES}

In this paper, the well-posedness of a wave equation with a nonlinear dynamic boundary condition and nonlinear Neuman-type feedback is proved, in a variational framework. Using a proportional feedback law, exponential stabilization is achieved under suitable assumptions.
In the linear case, an infinite-dimensional frequencydomain approach would be interesting to tackle the stabilization problem and obtain sharp conditions. Besides, adding an integral action to the feedback law should be considered. Also, the experimental device of [8] simulating drilling dynamics may be used to test the model in real experiments.

\section{REFERENCES}

[1] Delphine Bresch-Pietri and Miroslav Krstic. Output-feedback adaptive control of a wave PDE with boundary anti-damping. Automatica, 50(5):1407-1415, 2014.

[2] Haim Brezis. Functional analysis, Sobolev spaces and partial differential equations. Springer Science \& Business Media, 2010.

[3] Anthony Hastir, Federico Califano, and Hans Zwart. Well-posedness of infinite-dimensional linear systems with nonlinear feedback. Systems \& Control Letters, 128:19-25, 2019.

[4] Rhouma Mlayeh, Samir Toumi, and Lotfi Beji. Backstepping boundary observer based-control for hyperbolic PDE in rotary drilling system. Applied Mathematics and Computation, 322:66-78, 2018.

[5] Christophe Prieur, Sophie Tarbouriech, and Joao M. Gomes da Silva. Wave Equation With Cone-Bounded Control Laws. IEEE Transactions on Automatic Control, 61(11):3452-3463, 2016.

[6] Hector Ramirez, Hans Zwart, and Yann Le Gorrec. Stabilization of infinite dimensional port-Hamiltonian systems by nonlinear dynamic boundary control. Automatica, 85:61-69, 2017.

[7] Thiago G. Ritto, Christian Soize, and Rubens Sampaio. Non-linear dynamics of a drill-string with uncertain model of the bitrock interaction. International Journal of Non-Linear Mechanics, 44(8):865-876, 2009.

[8] Christophe Roman. Boundary control of a wave equation with indomain damping. PhD Thesis, Université Grenoble Alpes, 2018.

[9] Christophe Roman, Delphine Bresch-Pietri, Eduardo Cerpa, Christophe Prieur, and Olivier Sename. Backstepping control of a wave PDE with unstable source terms and dynamic boundary. IEEE Control Systems Letters, 2(3):459-464, 2018.

[10] Belem Saldivar, Sabine Mondi, and Juan Carlos vila Vilchis. The control of drilling vibrations: A coupled PDE-ODE modeling approach. International Journal of Applied Mathematics and Computer Science, 26(2):335-349, 2016.

[11] Ralph Edwin Showalter. Monotone operators in Banach space and nonlinear partial differential equations, volume 49. American Mathematical Soc., 2013.

[12] Andrey Smyshlyaev and Miroslav Krstic. Boundary control of an antistable wave equation with anti-damping on the uncontrolled boundary. Systems \& Control Letters, 58(8):617-623, 2009.

[13] Alexandre Terrand-Jeanne, Vincent Andrieu, Melaz Tayakout-Fayolle, and Valerie Dos Santos Martins. Regulation of Inhomogeneous Drilling Model With a P-I Controller. IEEE Transactions on Automatic Control, 65(1):58-71, 2020.

\section{APPENDIX}

We use the following existence result, which is an application of the Galerkin method and the Brouwer fixedpoint theorem in finite dimension - see [11, Lemma 2.1 and Theorem 2.1] for the proof.

Lemma 3. Let $E$ be a separable reflexive Banach space and $f \in E^{\prime}$. Assume $\Theta: E \rightarrow E^{\prime}$ verifies the following assumptions:

1) $\Theta$ is monotone i.e. $\left\langle\Theta\left(x_{1}\right)-\Theta\left(x_{2}\right), x_{1}-x_{2}\right\rangle_{E} \geq 0$ for all $x_{1}, x_{2} \in E$;

2) $\Theta$ is bounded i.e. $S \subset E$ bounded implies $\Theta(S)$ bounded in $E^{\prime}$;

3) For all $x_{1}, x_{2} \in E$, the scalar function $t \mapsto\left\langle\Theta\left(x_{1}+\right.\right.$ $\left.\left.t x_{2}\right), x_{2}\right\rangle_{E^{\prime}, E}$ is continuous.

If for some $\rho>0,\|x\|_{E}>\rho$ implies $\langle\Theta(x), x\rangle_{E^{\prime}, E}>$ $\langle f, x\rangle_{E^{\prime}, E}$, then there exists $x \in E$ such that $\Theta(x)=f$. 